Tôhoku Math. Journ.

25 (1973), 317-331.

\title{
THE GROWTH OF THE RESOLVENT AND HYPERINVARIANT SUBSPACES
}

\author{
Dedicated to Professor Masanori Fukamiya on his 60th birthday
}

\section{KOH-ICHI KITANO}

(Received November 6, 1972)

1. Introduction. Let $\mathfrak{X}$ (respectively, $\mathfrak{E}$ ) be a separable, infinite dimensional complex Banach (respectively, Hilbert) space. We denote by $\mathfrak{B}(\mathfrak{X})$ the algebra of all bounded linear operators acting in $\mathfrak{X}$. Let $T$ be a bounded linear operator on $\mathfrak{X}$. We call the bi-commutant of $T$ the set of operators commuting with all operators which commute with $T$. A closed subspace $\mathfrak{Y}$ of $\mathfrak{X}$ is said to be hyperinvariant for $T$ if $S \mathfrak{Y} \subset \mathfrak{V}$ for any operator $S$ that commutes with $T$.

The purpose of the present note is to show the existence of hyperinvariant subspaces for an operator $T$ with a spectrum condition and with a growth condition on the resolvent of $T$. The result will be considered as a generalization of those results on invariant subspaces in [2], [5]. In the case of a Hilbert space, such an operator, $T=A+B$, where $A$ is a normal operator with some spectrum condition and $B$ is a compact operator with some condition, has been studied in [2], [5].

Throughout this note, we denote by $\sigma(T), \sigma_{p}(T), \sigma_{c}(T), \sigma_{r}(T)$ and $\rho(T)$ the spectrum, the point spectrum, the continuous spectrum, the residual spectrum and the resolvent set of $T$, respectively (see Chap. VII of Part I of [3]). For any complex number $\lambda$ and a subset $E$ of the complex plane, we put $d(\lambda, E)=\inf _{z \in E}|\lambda-z|$. We understand a smooth arc to be such a one that has a continuous second derivative when parametrized with respect to arc length. We assume a Jordan curve $J$ is positively oriented and for a fixed $\lambda_{0}$ on $J$, where $J$ has a parametrization $\lambda=g(s)$ $(0 \leqq s \leqq l(J))$, in terms of arc length $s$ from $\lambda_{0}, g(0)=\lambda_{0}, g(s)=g(s+l(J))$, and $g(s)$ is continuous on $J$ and $g^{\prime}(s), g^{\prime \prime}(s)$ are continuous except points $\lambda_{k}=g\left(s_{k}\right), s_{k}<s_{k+1}, k=1,2, \cdots, n$ on $J$, where $l(J)$ denotes the whole length of $J$.

2. The existence of hyperinvariant subspaces for some operators. We denote by $J$, a Jordan curve, which consists of a finite number of rectifiable smooth arcs in the complex plane. The set of all operators $T \in \mathfrak{B}(\mathfrak{X})$ such that $\sigma(T) \subset J$ will be denoted by $\mathfrak{B}(\mathfrak{X} ; J)$, (it may well be the case 
that the spectrum separates the complex plane).

Let $T \in \mathfrak{B}(\mathfrak{X} ; J)$.

Definition 2.1. We say that the resolvent of $T$ has the growth condition $(C)$ near $J$ if

$$
\int_{0}^{\varepsilon} \log \log M(\delta) d \delta<\infty
$$

for some sufficiently small $\varepsilon>0$, where $M(\delta)=\sup _{d(\lambda, J) \geq \delta}\left\|(\lambda I-T)^{-1}\right\|$.

In [2], the resolvent is said to have polar growth of order $n$ (respectively, exponential growth of order $p$ ) near $J$ if $d(\lambda, J)^{n}\left\|(\lambda I-T)^{-1}\right\|$ (respectively, $\left.d(\lambda, J)^{p} \log \left\|(\lambda I-T)^{-1}\right\|\right), \lambda \notin J$ is bounded near $J$. If the resolvent has exponential growth of order $p$ (for any $p>0$ ) near $J$, then it has the growth condition $(C)$ near $J$. (Obviously, the resolvent has polar growth of order $n$ near $J$, then it has exponential growth of order $p$ for any $p>0$ ). This growth condition in Definition 2.1 plays an important role in the future. In this section we have the following result.

Theorem 2.2. Let $T \in \mathfrak{B}(\mathfrak{X} ; J)$ such that the resolvent of $T$ has the growth condition $(C)$ near $J$. If $\sigma(T)$ is not reduced to a single point, then $T$ has a proper closed hyperinvariant subspace.

We shall divide the proof into portions. The assertion follows immediately in the case that $\sigma(T)$ is disconnected. In fact, if $\sigma(T)$ is disconnected, let $\sigma_{0}$ be a proper open and closed subset of its spectrum $\sigma(T)$ and let $\Gamma_{0}$ be a contour enclosing $\sigma_{0}$ but no other point of $\sigma(T)-\sigma_{0}$ and lying in $\rho(T)$, then the spectral projection $E\left(\sigma_{0}\right)$ corresponding to $\sigma_{0}$,

$$
E\left(\sigma_{0}\right)=\frac{1}{2 \pi i} \int_{\Gamma_{0}}(\lambda I-T)^{-1} d \lambda
$$

is an idempotent distinct from 0 and $I$ which commutes with every operator commuting with $T$ and $E\left(\sigma_{0}\right)$ would define a proper closed hyperinvariant subspace $E\left(\sigma_{0}\right) \mathfrak{X}$ of $\mathfrak{X}$ with respect to $T$ (see Chap. XI of [11]). Therefore we have only to show that there can exist no operator $T \in$ $\mathfrak{B}(\mathfrak{X} ; J)$ such that the resolvent of $T$ has the growth condition $(C)$ near $J$ and $\sigma(T)$ is connected subarc of $J$ and $\mathfrak{X}$ admits no proper closed hyperinvariant subspace under $T$.

Now we need the following generalization of Levinson's theorem [7, Lemma 27.1, p. 135].

Lemma 2.3. (Ljubič and Macaev [8]). Suppose that $M(\delta)$ is nonincreasing function $(\delta>0)$ satisfying the following conditions, $M(\delta) \rightarrow \infty$ as $\delta \rightarrow 0$ and 


$$
\int_{0}^{\varepsilon} \log \log M(\delta) d \delta<\infty
$$

for some sufficiently small $\varepsilon>0$, and suppose that $J_{0}$ is a smooth arc in the complex plane, beginning at some point $z_{0}$. Then there exists a function analytic except for $z=z_{0}$ satisfying the following conditions:

(i) $|f(z)| \leqq 1 / M(\delta)$ along some beak $\Gamma$ (Fig. 1) formed by two smooth

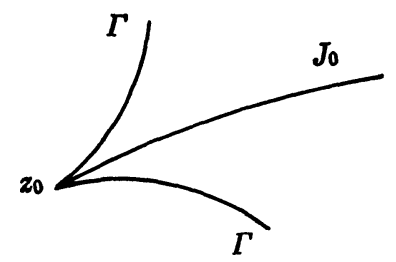

Fig. 1

arcs tangent to $J_{0}$ on opposite sides at the point $z_{0}$, where $\delta=d\left(z, J_{0}\right)$;

(ii) $f(z)$ is continuous on the inside and on the beak $\Gamma$;

(iii) $f(z) \neq 0($ for $z \neq \infty), f(\infty)=0$.

Using Lemma 2.3 we have the followings. For any point $\mu \in J$, which is not a singular point of $J$, we denote $f_{\mu}^{+}(\lambda)$ and $f_{\mu}^{-}(\lambda)$ analytic functions whose existence is assured by Lemma 2.3, constructed with respect to the majorant $M(\delta)$ and curves $J_{\mu}^{+}$and $J_{\mu}^{-}$respectively $\left(J_{\mu}^{+}\right.$and $J_{\bar{\mu}}^{-}$are the Jordan curve $J$ issuing from the point $\mu \in J$ respectively in positive and negative directions). We denote corresponding beaks by $\Gamma_{\mu}^{+}$ and $\Gamma_{\mu}^{-}$respectively. Let $\Delta=[\alpha, \beta]$ be any segment of the curve $J$, where $\alpha, \beta$ are not singular points of $J$ ( $\alpha$ precedes $\beta$ in a positive direction along $J)$. Put

$$
F_{\Delta}(\lambda)=f_{\alpha}^{+}(\lambda) f_{\beta}^{-}(\lambda)
$$

and denote by $\Gamma_{\Delta}$ the contour obtained by joining $\Gamma_{\alpha}^{+}$and $\Gamma_{\bar{\beta}}^{-}$by means of any two simple curves, which consists of a finite number of rectifiable smooth arcs (Fig. 2). The function $F_{\Delta}(\lambda)$ is evidently analytic on the

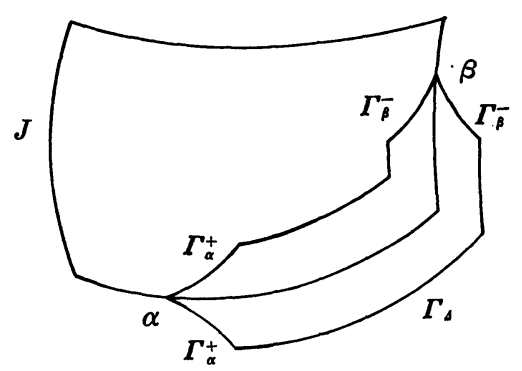

Fig. 2 
inside of $\Gamma_{\Delta}$, continuous on the inside and on $\Gamma_{\Delta}$ and does not vanish on the inside of $\Gamma_{\Delta}$. From the construction following estimates hold

$$
\begin{aligned}
& F_{\Delta}(\lambda)=O(1 / M(d(\lambda, J))) \text { for } \lambda \longrightarrow \alpha, \lambda \in \Gamma_{\Delta}, \\
& F_{\Delta}(\lambda)=O(1 / M(d(\lambda, J))) \text { for } \lambda \longrightarrow \beta, \lambda \in \Gamma_{\Delta},
\end{aligned}
$$

where $O(\cdot)$ is Landau's symbol.

LEMMA 2.4. Let $T \in \mathfrak{B}(\mathfrak{X} ; J)$ such that $\sigma(T)$ is a connected set (not a single point) and the resolvent of $T$ has the growth condition $(C)$ near $J$. For each pair of points $\alpha, \beta$ where $\alpha$ precedes $\beta$ in a positive direction along $J$ and they are not singular points of $J$, we put

$$
\begin{aligned}
\mathfrak{S}(\alpha, \beta)= & \left\{x ; x \in \mathfrak{X},(\lambda I-T)^{-1} x\right. \text { is continuable to a function which is } \\
& \text { analytic on } \rho(T) \cup\{\text { the arc }(\alpha, \beta)\}\}, \\
\widetilde{S}(\alpha, \beta)= & \left\{x ; x \in \mathfrak{X},(\lambda I-T)^{-1} x\right. \text { is continuable to a function which is } \\
& \text { analytic on } \rho(T) \cup\{\text { the arc }(\beta, \alpha)\}\} .
\end{aligned}
$$

Then both $\Im(\alpha, \beta)$ and $\widetilde{\subseteq}(\alpha, \beta)$ are closed subspaces of $\mathfrak{X}$, invariant under $T$.

Proof. Because both of the invariance and the linearity of $\mathfrak{S}(\alpha, \beta)$ are plain, we have only to prove that $\mathfrak{S}(\alpha, \beta)$ is closed. Let $x_{n} \in \mathfrak{S}(\alpha, \beta)$, $(n=1,2,3, \cdots)$ and $x_{n} \rightarrow x$ as $n \rightarrow \infty$. Let $R\left(\lambda, T: x_{n}\right)$ denote the analytic continuation of $(\lambda I-T)^{-1} x_{n}$, then for every positive number $\varepsilon$, let $\Delta=$ $[\alpha+\varepsilon, \beta-\varepsilon]$ in above argument. Then $R\left(\lambda, T: x_{n}\right)$ are analytic on the inside of $\Gamma_{\Delta}$. Here we define the function such that

$$
g_{n}(\lambda)=\left\{\begin{array}{r}
F_{\Delta}(\lambda)(\lambda-\alpha-\varepsilon)(\lambda-\beta+\varepsilon) R\left(\lambda, T: x_{n}\right) \\
\text { if } \lambda \neq \alpha+\varepsilon \text { and } \lambda \neq \beta-\varepsilon, \\
0 \quad \text { if } \lambda=\alpha+\varepsilon \text { or } \lambda=\beta-\varepsilon,
\end{array}\right.
$$

then $g_{n}(\lambda)$ are analytic on the inside of $\Gamma_{\Delta}$ and strongly continuous on $\Gamma_{\Delta}$, which follows from estimates of $F_{\Delta}(\lambda)$. By the maximum modulus principle, $\left\{g_{n}(\lambda)\right\}$ is a uniform Cauchy sequence with respect to $\lambda$, hence the limit function $g_{0}(\lambda)$ is analytic on the inside of $\Gamma_{\Delta}$ and so

$$
x_{\infty}(\lambda)=g_{0}(\lambda)\left\{F_{\Delta}(\lambda)(\lambda-\alpha-\varepsilon)(\lambda-\beta+\varepsilon)\right\}^{-1}
$$

is also analytic on the inside of $\Gamma_{\Delta}$. It follows that $(\lambda I-T)^{-1} x$ has an analytic continuation to a neighbourhood of the arc $(\alpha+\varepsilon, \beta-\varepsilon)$ for any sufficiently small $\varepsilon>0$, and hence to a neighbourhood of the arc $(\alpha, \beta)$. Thus $x \in \mathfrak{S}(\alpha, \beta)$. The assertion for $\widetilde{\Im}(\alpha, \beta)$ will be proved in just the same way. The proof of Lemma 2.4 is now completed.

LEMMA 2.5. Let $T, \Im(\alpha, \beta)$ and $\widetilde{\Im}(\alpha, \beta)$ be the same as in Lemma 
2.4 and $\tilde{\Delta}=[\beta, \alpha]$. For any vector $x \in \mathfrak{X}$, the function $g(\lambda)$ is defined such that

$$
g(\lambda)= \begin{cases}F_{\widetilde{\tau}}(\lambda)(\lambda-\alpha)(\lambda-\beta)(\lambda I-T)^{-1} x & \text { if } \lambda \in \Gamma_{\Delta}-\{\alpha, \beta\}, \\ 0 & \text { if } \lambda=\alpha \text { or } \lambda=\beta .\end{cases}
$$

If $b(z)$ is any numerical-valued function analytic in and continuous on $|z| \leqq 1$ and if $\tau$ is the conformal mapping from the inside of $\Gamma_{\widetilde{a}}$ to the unit disc, then the contour integral

$$
y=\int_{C_{0}} b(z) g\left(\tau^{-1}(z)\right) d z
$$

belongs to the space $\mathfrak{S}(\alpha, \beta)$, where $C_{0}$ denotes the boundary of the unit disc (suppose that $C_{0}$ is positively oriented). Moreover, unless $x$ belongs to the space $\widetilde{\Xi}(\alpha, \beta)$ there exists a function $b(z)$ analytic in and continuous on $|z| \leqq 1$ such that the vector $y$ defined by $\left(^{*}\right)$ is non-zero.

Proof. Clearly, the function $g\left(\tau^{-1}(z)\right)$ is continuous on $C_{0}$. Using the resolvent equation, we have

$$
(\mu I-T)^{-1} g(\lambda)=(\mu-\lambda)^{-1} g(\lambda)-(\mu-\lambda)^{-1} F_{\check{\Delta}}(\lambda)(\lambda-\alpha)(\lambda-\beta)(\mu I-T)^{-1} x
$$

for $\mu \in \rho(T) \cap \operatorname{Ext} \Gamma_{\widetilde{a}}$, where $\operatorname{Ext} \Gamma_{\widetilde{\lrcorner}}$ denotes the outside of $\Gamma_{\widetilde{\lrcorner}}$, thus

$$
\begin{aligned}
(\mu I-T)^{-1} y= & \int_{C_{0}} \frac{b(z) g\left(\tau^{-1}(z)\right)}{\mu-\tau^{-1}(z)} d z \\
& -\int_{C_{0}} \frac{b(z) F_{\widetilde{u}}\left(\tau^{-1}(z)\right)\left(\tau^{-1}(z)-\alpha\right)\left(\tau^{-1}(z)-\beta\right)(\mu I-T)^{-1} x}{\mu-\tau^{-1}(z)} d z
\end{aligned}
$$

for the vector $y$ of $\left(^{*}\right)$. By Cauchy's theorem, the second term is zero. Therefore we have

$$
(\mu I-T)^{-1} y=\int_{c_{0}} \frac{b(z) g\left(\tau^{-1}(z)\right)}{\mu-\tau^{-1}(z)} d z
$$

for the vector $y$ of $(*)$. Since the final expression of $\left({ }^{*}\right)$ is plainly analytic on the outside of $\Gamma_{\widetilde{a}}$, it follows that $y \in \mathfrak{S}(\alpha, \beta)$. Next, we suppose that the vector $y$ defined by $\left(^{*}\right)$ is zero for each $b(z)$ which is analytic in and continuous on $|z| \leqq 1$, i.e., for all such $b(z)$

$$
\int_{C_{0}} b(z) g\left(\tau^{-1}(z)\right) d z=0
$$

Therefore the vector-valued function $g\left(\tau^{-1}(z)\right)$ defined on $C_{0}$ must be the boundary value of a vector-valued function analytic in and continuous on $|z| \leqq 1$. Therefore $g(\lambda)$ must be analytically continuable to the inside of $\Gamma_{\check{\Delta}}$. Thus $(\lambda I-T)^{-1} x$ must be continuable across the arc $(\beta, \alpha)$, i.e., 
$x \in \widetilde{\Phi}(\alpha, \beta)$. The proof of Lemma 2.4 is now completed.

Let $T, \mathscr{S}(\alpha, \beta), \widetilde{\Phi}(\alpha, \beta), \Delta, \widetilde{\Delta}$ and $C_{0}$ be the same as above. Let $\tau$ (respectively, $\nu$ ) be a conformal mapping from the inside of $\Gamma_{\widetilde{\Omega}}$ (respectively, $\left.\Gamma_{\lrcorner}\right)$to the unit disc. Now we define the function $g_{\tilde{a}}(\lambda)$ such that

$$
g_{\tilde{\Delta}}(\lambda)= \begin{cases}F_{\tilde{\alpha}}(\lambda)(\lambda-\alpha)(\lambda-\beta)(\lambda I-T)^{-1} & \text { if } \lambda \in \Gamma_{\tilde{\Delta}}-\{\alpha, \beta\}, \\ 0 & \text { if } \lambda=\alpha \text { or } \lambda=\beta .\end{cases}
$$

Let $b_{\widetilde{\tau}}(z)$ be a function analytic in and continuous on $|z| \leqq 1$ such that the contour integral

$$
\int_{C_{0}} b_{\widetilde{\jmath}}(z) g_{\widetilde{u}}\left(\tau^{-1}(z)\right) x d z
$$

is non-zero for some vector $x \notin \tilde{S}(\alpha, \beta)$. In just the same way we can define $g_{\Delta}(\lambda)$ and $b_{\Delta}(z)$ with respect to $\Delta$ instead of $\tilde{\Delta}$.

LeMma 2.6. If we put

$$
S=\int_{C_{0}} b_{\tilde{\Delta}}(z) g_{\tilde{\Delta}}\left(\tau^{-1}(z)\right) d z \text { and } W=\int_{c_{0}} b_{\lrcorner}(z) g_{\Lambda}\left(\nu^{-1}(z)\right) d z,
$$

then $\overline{S X}, \overline{W X}$, where bar denotes the closure, are hyperinvariant subspaces under $T$ and

$$
\{0\} \subset \overline{S \mathfrak{X}} \subset \Im(\alpha, \beta), \quad\{0\} \subset \overline{W \mathfrak{X}} \subset \widetilde{\Phi}(\alpha, \beta) .
$$

PRoof. From the proof of preceding Lemmas, the last assertions follows. Let $A$ be any operator in $\mathfrak{B}(\mathfrak{X})$ such that $A T=T A$. Since both operators $S, W$ are elements of the bi-commutant of $T$, it follows

$$
\begin{aligned}
A(\overline{S \mathfrak{X}}) \subset \overline{A S \mathfrak{X}} & =\overline{S A \mathfrak{X}} \subset \overline{S \mathfrak{X}}, \\
A(\overline{W \mathfrak{X}}) \subset \overline{A W \mathfrak{X}} & =\overline{W A \mathfrak{X}} \subset \overline{W \mathfrak{X}},
\end{aligned}
$$

The proof of Lemma 2.6 is now completed.

Proof of Theorem. Suppose that $T$ is an operator satisfying the hypotheses of Lemma 2.4. By Lemma 2.4 and Lemma 2.6, we have only to show that $\subseteq(\alpha, \beta)$ and $\widetilde{\varsigma}(\alpha, \beta)$ are non-trivial. We may assume $\sigma(T)$ lies on both arcs $(\alpha, \beta)$ and $(\beta, \alpha)$, because we can choose the pair of points $\alpha, \beta$ arbitrary on $J$. This implies $\mathfrak{S}(\alpha, \beta) \neq \mathfrak{X}$ and $\widetilde{\subseteq}(\alpha, \beta) \neq \mathfrak{X}$. Thus we have only to show that $\subseteq(\alpha, \beta) \neq\{0\}$ and $\widetilde{\varsigma}(\alpha, \beta) \neq\{0\}$. By Lemma 2.5, $\subseteq(\alpha, \beta) \neq \mathfrak{X}, \widetilde{\subseteq}(\alpha, \beta) \neq \mathfrak{X}$ imply $\widetilde{\subseteq}(\alpha, \beta) \neq\{0\}, \mathscr{S}(\alpha, \beta) \neq\{0\}$ respectively. Since both $\mathfrak{S}(\alpha, \beta), \widetilde{\subseteq}(\alpha, \beta)$ are non-trivial and by definitions in Lemma 2.6 $S \neq 0, W \neq 0$. Thus from Lemma 2.6 the assertion follows.

3. Perturbation and hyperinvariant subspaces. In the sequel $J$ will stand a finite union of simple rectifiable smooth ares in the complex plane 
(not separating the complex plane). Instead of $\mathfrak{X}$ we consider a Hilbert space $\mathfrak{S}$. Let $C_{\infty}$ be the set of all compact operators acting in $\mathfrak{S}$. Let $T \in C_{\infty}$. We denote by $C_{\omega}$ the class of compact operators for which

$$
\|T\|_{\omega}=\sum_{n=1}^{\infty} \mu_{n}(T) / n<\infty,
$$

where $\mu_{1}(T), \mu_{2}(T), \cdots, \mu_{n}(T), \cdots$ are the eigenvalues of $\left(T^{*} T\right)^{1 / 2}$ arranged in decreasing order and repeated according to multiplicity (see $[4, \S 15$ of Chap. III]) and the class $C_{p}$ is the set of all compact operators such that $\|T\|_{p}=\left\{\sum_{n=1}^{\infty} \mu_{n}(T)^{p}\right\}^{1 / p},(1 \leqq p<\infty)$ is finite (see [3,§9 of Chap. $\mathrm{XI}])$. It is easy to see that $C_{p} \subset C_{\omega}$ for arbitrary $p(1 \leqq p<\infty)$. In fact, from Hölder's inequality it follows that

$$
\|T\|_{\omega} \leqq\left\{\sum_{n} 1 / n^{q}\right\}^{1 / q}\left\{\sum_{n} \mu_{n}(T)^{p}\right\}^{1 / p},
$$

where $1 / p+1 / q=1$. The class $C_{\omega}$ was introduced in the paper of V.I. Macaev [9]. To an operator $T \in C_{\infty}$ we associate the sequences $\lambda_{1}(T)$, $\lambda_{2}(T), \cdots, \lambda_{n}(T), \cdots$ of eigenvalues of $T$ numbered according to decreasing values of their moduli and repeated according to multiplicity and the function $n(t, T)$ (respectively, $\gamma(t, T)$ ), for $t>0$, giving the number of terms of the sequence $\lambda_{n}(T)$ (respectively, $\left.\mu_{n}(T)\right), n=1,2, \cdots, n, \cdots$, exceeding $1 / t$ in modulus.

The purpose of this section is to show that the following theorem holds.

THEOREM 3.1. Let $T$ be an operator in $\mathfrak{B}(\mathfrak{W})$. Write $T=A+B$, where $T$ is the sum of a normal operator $A$, whose spectrum lies on $J$ and a compact operator $B$ which belongs to the class $C_{\omega}$. If $\sigma(T)$ is not reduced to a single point, then $T$ has a proper closed hyperinvariant subspace in $\mathfrak{S}$.

On the existence of invariant subspace, in [5] the author has proved in the case that the perturbing term belongs to one of the classes $C_{p}(1 \leqq p<\infty)$. In $\S 3$ of [2], Apostol studies operators of the form $A+B$, which the resolvent of $A$ has polar growth of order $n$ near $J$ and $B \in C_{p}(1 \leqq p<\infty)$.

REMARK. In the above theorem if $\sigma(T)$ is a single point, then $T$ has a proper closed invariant subspace. In fact, since the operator $T$ may be translated, we may assume without loss of generality that $\sigma(T)=\{0\}$, from Lemma 2.3 of [5] it follows that the Hilbert space admits a proper closed invariant subspace under $T$, using Aronszajn-Smith theorem [1].

We shall use the following known result. 
Lemma 3.2. (see [4, $\S 5$ of Chap. I]) Let $T$ be as above (in the theorem). Then any point $\lambda$ with $\lambda \notin \sigma(A)(\subset J)$ is either $\lambda \in \rho(T)$ or an eigenvalue with finite multiplicity of $T$.

Lemma 3.3. If $T$ has an eigenvalue with finite multiplicity, then $\mathfrak{S}$ admits a proper closed subspace which is hyperinvariant under $T$.

Proof. Let $\lambda_{0}$ be an eigenvalue with $n$ multiplicity and $S T=T S$ $(S \in \mathfrak{B}(\mathfrak{E}))$. Then the eigensubspace of $T$ corresponding to $\lambda_{0}$ is an $n$ dimensional subspace invariant under $S$. In fact, since

$$
\left(T-\lambda_{0} I\right) S\left\{\left(T-\lambda_{0} I\right)^{-1}(0)\right\}=S\left(T-\lambda_{0} I\right)\left\{\left(T-\lambda_{0} I\right)^{-1}(0)\right\}=S(0)=\{0\} .
$$

Therefore we have

$$
S\left\{\left(T-\lambda_{0} I\right)^{-1}(0)\right\} \subset\left(T-\lambda_{0} I\right)^{-1}(0) .
$$

The proof of Lemma 3.3 is now completed.

The assertion in Theorem follows from Lemma 3.3 and an argument in $\S 2$ in each case that one of the following condition is satisfied:

$$
\text { (i) } \sigma(T)-\sigma(A) \neq \varnothing ; \quad \text { (ii) } \sigma(T) \text { is disconnected . }
$$

Therefore we have only to show that there can exist no operator $T=$ $A+B$, where $A$ is normal such that $\sigma(A) \subset J, B \in C_{\omega}$ and $\sigma(T)(\subset \sigma(A))$ is a connected subarc of $J$ and $\mathscr{S}$ admits no proper closed hyperinvariant subspace under $T$.

In the sequel, we shall show a growth condition of the resolvent of $T$ near its spectrum $\sigma(T)$. If $S$ has finite dimensional range, $S=P S$, where $P$ is the orthogonal projection on the range of $S$. Thus $S^{*}=S^{*} P$, so that $S^{*}$ also has finite dimensional range. Let $\mathfrak{S}_{0}$ be a finite dimensional space including both the range of $S$ and the range of $S^{*}$. Then plainly, $\mathfrak{S}_{0}$ is invariant under $S$ and $S^{*}$, and since $\left(S \mathfrak{S}_{0}^{\perp}, x\right)=\left(\mathfrak{S}_{0}^{\perp}, S^{*} x\right)=0$ for all $x \in \mathfrak{S}_{\mathfrak{C}}$, we have $S \mathfrak{S}_{0}^{\perp}=\{0\}$ and similarly $S^{*} \mathfrak{S}_{0}^{\perp}=\{0\}$. We denote by $\Phi=\left\{\varphi_{1}, \varphi_{2}, \cdots, \varphi_{n}\right\}$ an orthonormal basis of $\mathscr{S}_{0}$ and define an $n \times n$ matrix $S_{(n)}=\left(a_{i j}\right)_{1 \leqq i, j \leqq n}$ by the following relation

$$
S \varphi_{j}=\sum_{i=1}^{n} a_{i j} \varphi_{i} \quad(j=1,2, \cdots, n) .
$$

Thus $S_{(n)}$ is the matrix of the operator $S \mid \mathfrak{S}_{0}$ (= the restriction of $S$ to $\mathfrak{S}_{0}$ ) with respect to the basis $\Phi$.

We define

$$
D_{1}(S)=\operatorname{det}\left(I_{(n)}-S_{(n)}\right)
$$

the determinant of $I_{(n)}-S_{(n)}$, where $I_{(n)}$ is the $n \times n$ identity matrix. 
Now, we denote by $K=\left(k_{i j}\right)_{1 \leq i, j \leq n}$ the cofactor matrix of $I_{(n)}-S_{(n)}$ i.e., $k_{i j}$ is the $(j, i)$ cofactor of $I_{(n)}-S_{(n)}$. We have

$$
\left(I_{(n)}-S_{(n)}\right) K=K\left(I_{(n)}-S_{(n)}\right)=D_{1}(S) I_{(n)} .
$$

We define an $n \times n$ matrix $\left(h_{i j}\right)_{1 \leq i, j \leq n}=S_{(n)} K$ and an operator $H$ on $\mathfrak{E}$ such that

$$
H x=\sum_{j=1}^{n} \sum_{i=1}^{n} h_{i j}\left(x, \varphi_{j}\right) \varphi_{i} \quad \text { for } \quad x \in \mathfrak{Q} .
$$

Then we have $H \mathfrak{S}_{0} \subset \mathfrak{S}_{0}$ and $H \mathfrak{S}_{0}^{\perp}=\{0\}$. Therefore the restriction $H \mid \mathfrak{S}_{0}$ of $H$ to $\mathfrak{S}_{0}$ is the operator whose matrix representation with respect to the basis $\Phi$ is the matrix $\left(h_{i j}\right)$. For operators $\left(D_{1}(S) I+H\right)(I-S)$ and $(I-S) \times$ $\left(D_{1}(S) I+H\right)$, the subspaces $\mathfrak{S}_{0}$ and $\mathfrak{S}_{0}^{\perp}$ are invariant and it follows that by the above relations

$$
\left(D_{1}(S) I+H\right)(I-S) x=(I-S)\left(D_{1}(S) I+H\right) x=D_{1}(S) x
$$

for any $x \in \mathfrak{S}_{0}^{\perp}$. Moreover, since

$$
D_{1}(S) I_{(n)}+S_{(n)} K=\left(I_{(n)}-S_{(n)}\right) K+S_{(n)} K=K,
$$

we have the matrix relations

$$
\begin{aligned}
& \left(D_{1}(S) I_{(n)}+S_{(n)} K\right)\left(I_{(n)}-S_{(n)}\right) \\
& \quad=\left(I_{(n)}-S_{(n)}\right)\left(D_{1}(S) I_{(n)}+S_{(n)} K\right)=D_{1}(S) I_{(n)},
\end{aligned}
$$

i.e., by restriction each operator to $\mathfrak{S}_{0}$ we have the last above relations with respect to the basis $\Phi$. Thus we have the following equations of operators on the subspace $\mathfrak{S}_{0}$,

$$
\begin{aligned}
& \left(D_{1}(S) I\left|\mathscr{S}_{0}+H\right| \mathscr{S}_{0}\right)\left(I\left|\mathscr{S}_{0}-S\right| \mathscr{S}_{0}\right) \\
& \quad=\left(I\left|\mathfrak{S}_{0}-S\right| \mathscr{S}_{0}\right)\left(D_{1}(S) I\left|\mathscr{S}_{0}+H\right| \mathscr{S}_{0}\right)=D_{1}(S) I \mid \mathfrak{S}_{0},
\end{aligned}
$$

therefore we obtain the operator relations on $\mathfrak{S}$,

$$
\left(D_{1}(S) I+H\right)(I-S)=(I-S)\left(D_{1}(S) I+H\right)=D_{1}(S) I .
$$

So that $(I-S)^{-1}$ exists, when $D_{1}(S) \neq 0$, i.e., $1 \notin \sigma(S)$,

$$
(I-S)^{-1}=I+D_{1}(S)^{-1} H \text {. }
$$

Now, we define

$$
D_{2}(S)=D_{1}(S)\left((I-S)^{-1} x, y\right)
$$

for unit vectors $x, y$ in $\mathfrak{S}$.

LEMma 3.4. Let $S$ be a finite dimensional operator, $1 \notin \sigma(S)$, and let $D_{1}(S)$ and $D_{2}(S)$ be as above. Then we have the followings,

$$
\log \left|D_{1}(S)\right| \leqq \sum_{j=1}^{n} \log \left(1+\mu_{j}(S)\right)
$$




$$
\log \left|D_{2}(S)\right| \leqq \sum_{j=1}^{n} \log \left(1+\mu_{j}(S)\right)+3 \log \left(3+\mu_{1}(S)\right) .
$$

Proof. To prove the second assertion, it is sufficient to establish the corresponding inequality for the operator obtained by restricting $I+$ $D_{1}(S)^{-1} H$ to the subspace $\mathscr{S}_{0}$. In fact, since $H \mathfrak{S}_{0}^{\perp}=\{0\}$, the restriction $(I-S)^{-1}$ to $\mathfrak{S}_{0}^{\perp}$ is $I \mid \mathfrak{S}_{0}^{\perp}$ and the second assertion follows from the first assertion. Now, the matrix representation of $\left(I+D_{1}(S)^{-1} H\right) \mid \mathfrak{S}_{0}$ with respect to the basis $\Phi$ of $\mathfrak{S}_{0}$ is $I_{(n)}+D_{1}(S)^{-1} S_{(n)} K$ and we have

$$
\left(I+D_{1}(S)^{-1} H\right) \varphi_{j}=D_{1}(S)^{-1}\left(D_{1}(S) I+H\right) \varphi_{j}=D_{1}(S)^{-1} \sum_{i=1}^{n} k_{i j} \varphi_{i}
$$

$(j=1,2, \cdots, n)$. Thus we have

$$
\left(\left(D_{1}(S) I+H\right) \varphi_{j}, \varphi_{i}\right)=k_{i j} \text {. }
$$

Given any unit vectors $x, y$ in $\mathfrak{S}_{0}$, we may put $x=x_{1} \varphi_{1}+\cdots+x_{n} \varphi_{n}$ and $y=y_{1} \varphi_{1}+\cdots+y_{n} \varphi_{n}$, where $\sum_{i=1}^{n}\left|x_{i}\right|^{2}=\sum_{i=1}^{n}\left|y_{i}\right|^{2}=1$. Then

$$
\begin{aligned}
D_{1}(S)\left((I-S)^{-1} x, y\right)=\left(\left(D_{1}(S) I+H\right) x, y\right) \\
=\sum_{i, j=1}^{n} k_{i j} x_{j} \bar{y}_{i}=-\operatorname{det}\left[\begin{array}{lll}
0 & \bar{y}_{1} & \bar{y}_{2} \\
x_{1} & & \\
x_{2} & I_{(n)} \\
\vdots & & \\
x_{n} &
\end{array}\right] .
\end{aligned}
$$

On the other hand, since we have the relation (by Lemma 16 of Chap. XI. 9 of [3]),

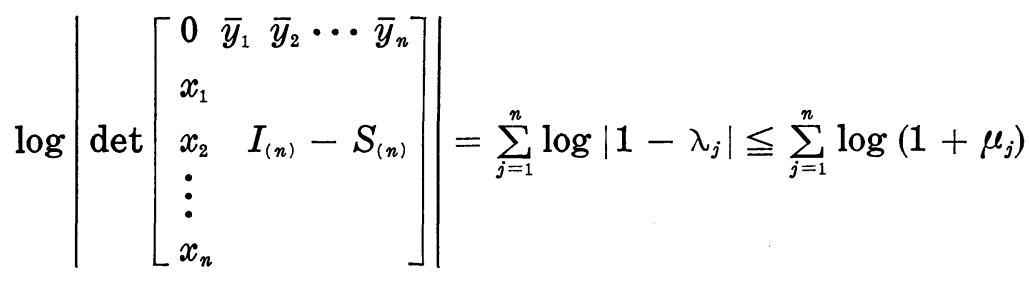

with the eigenvalues $\left\{\lambda_{j}\right\}$ (respectively, $\left\{\mu_{j}\right\}$ ) of

$$
S_{0}=\left[\begin{array}{lll}
1 & \bar{y}_{1} & \bar{y}_{2} \cdots \bar{y}_{n} \\
x_{1} & \\
x_{2} & S_{(n)} \\
\vdots & \\
x_{n} &
\end{array}\right]
$$

(respectively, $\left.\left(S_{0}{ }^{*} S_{0}\right)^{1 / 2}\right)$. If we put 


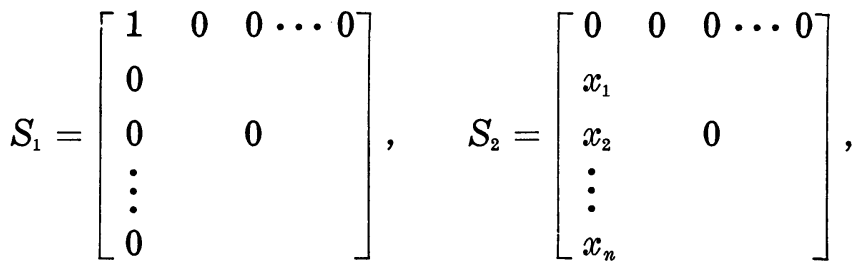

$$
\begin{aligned}
& S_{3}=\left[\begin{array}{cccc}
0 & \bar{y}_{1} & \bar{y}_{2} \cdots & \bar{y}_{n} \\
0 & & \\
0 & 0 \\
\vdots & & \\
0 & &
\end{array}\right], \quad S_{4}=\left[\begin{array}{ccl}
0 & 0 & 0 \cdots 0 \\
0 & & \\
0 & S_{(n)} \\
\vdots & & \\
0 & &
\end{array}\right] \text {, }
\end{aligned}
$$

then $S_{0}=S_{1}+S_{2}+S_{3}+S_{4}$. By virtue of corollary 3 of Chap. XI. 9 of [3], for $\mu_{j}\left(S_{0}\right)$ we have the followings

$$
\begin{aligned}
& \mu_{1}\left(S_{0}\right) \leqq \mu_{1}\left(S_{1}\right)+\mu_{1}\left(S_{2}\right)+\mu_{1}\left(S_{3}\right)+\mu_{1}\left(S_{4}\right)=3+\mu_{1}(S), \\
& \mu_{2}\left(S_{0}\right) \leqq \mu_{1}\left(S_{1}\right)+\mu_{1}\left(S_{2}\right)+\mu_{2}\left(S_{3}\right)+\mu_{1}\left(S_{4}\right)=2+\mu_{1}(S) \text {, } \\
& \mu_{3}\left(S_{0}\right) \leqq \mu_{1}\left(S_{1}\right)+\mu_{2}\left(S_{2}\right)+\mu_{2}\left(S_{3}\right)+\mu_{1}\left(S_{4}\right)=1+\mu_{1}(S), \\
& \mu_{4}\left(S_{0}\right) \leqq \mu_{2}\left(S_{1}\right)+\mu_{2}\left(S_{2}\right)+\mu_{2}\left(S_{3}\right)+\mu_{1}\left(S_{4}\right)=\mu_{1}(S) \text {, } \\
& \mu_{j}\left(S_{0}\right) \leqq \mu_{j-3}(S) \text {, } \\
& \mu_{n+1}\left(S_{0}\right) \leqq \mu_{n-2}(S),
\end{aligned}
$$

therefore we have

$$
\begin{aligned}
\sum_{j=1}^{n+1} \log \left(1+\mu_{j}\left(S_{0}\right)\right) & \leqq \sum_{j=1}^{n-2} \log \left(1+\mu_{j}(S)\right)+\log \left(4+\mu_{1}(S)\right)\left(3+\mu_{1}(S)\right)\left(2+\mu_{1}(S)\right) \\
& \leqq \sum_{j=1}^{n} \log \left(1+\mu_{j}(S)\right)+3 \log \left(3+\mu_{1}(S)\right)
\end{aligned}
$$

Thus the second assertion follows. The first assertion follows immediately, in fact

$$
\log \left|D_{1}(S)\right|=\sum_{j=1}^{n} \log \left|1-\lambda_{j}(S)\right| \leqq \sum_{j=1}^{n} \log \left(1+\mu_{j}(S)\right)
$$

This completes the proof of Lemma 3.4.

Now without loss of generality, we may assume that $\|B\| \leqq 1 / 2$. For any sufficiently small number $\delta>0$, let $P$ be the projection to the spectral subspace of $\left(B^{*} B\right)^{1 / 2}$ relative to $(\delta, 1 / 2]$ and $B_{1}=B P, B_{2}=B(I-P)$. Since $A$ is normal, we have $\left\|(\lambda I-A)^{-1}\right\| \leqq\{d(\lambda, \sigma(A))\}^{-1}$. Thus by $\left\|B_{2}(\lambda I-A)^{-1}\right\| \leqq \delta / d(\lambda, \sigma(A))$, 


$$
\begin{aligned}
\left\|\left(\lambda I-A-B_{2}\right)^{-1}\right\| & =\left\|(\lambda I-A)^{-1}\left\{I-B_{2}(\lambda I-A)^{-1}\right\}^{-1}\right\| \\
& \leqq\left\|(\lambda I-A)^{-1}\right\|\left\|\left\{I-B_{2}(\lambda I-A)^{-1}\right\}^{-1}\right\| \\
& \leqq\{d(\lambda, \sigma(A))-\delta\}^{-1} \\
& \leqq 1 / \delta,
\end{aligned}
$$

for $\lambda$ with $d(\lambda, \sigma(A)) \geqq 2 \delta$. We have

$$
\begin{aligned}
& \log \left\|(\lambda I-T)^{-1}\right\| \\
& \quad \leqq \log \left\|\left(\lambda I-A-B_{2}\right)^{-1}\right\|+\log \left\|\left\{I-B_{1}\left(\lambda I-A-B_{2}\right)^{-1}\right\}^{-1}\right\| .
\end{aligned}
$$

Now, to obtain an estimate of the second term in the sequel, extending the method for estimating the resolvent of a compact operator in [10], we shall show a growth condition of the resolvent of $T$ near its spectrum $\sigma(T)(\subset \sigma(A))$. Let $D_{k}(\lambda)=D_{k}\left(B_{1}\left(\lambda I-A-B_{2}\right)^{-1}\right)$, for $k=1$, 2. Let $z=$ $\tau(\lambda)$ be the conformal mapping of the domain $\{\lambda ; d(\lambda, \sigma(A)) \geqq 2 \delta\}$ onto the disc of radius $r$ with center at the origin and $\tau(\infty)=0$. We put $n(t)=$ $n\left(t, B_{1}\left(\tau^{-1}(z) I-A-B_{2}\right)^{-1}\right)$, by Jensen's formula [6, Theorem 5, p. 14]

$$
\begin{aligned}
\int_{0}^{r} \frac{n(t)}{t} d t= & \frac{1}{2 \pi} \int_{0}^{2 \pi} \log \left|D_{1}\left(\tau^{-1}\left(r e^{i \theta}\right)\right)\right| d \theta-\log \left|D_{1}\left(\tau^{-1}(0)\right)\right| \\
= & -\frac{1}{2 \pi} \int_{0}^{2 \pi} \log ^{-}\left|D_{1}\left(\tau^{-1}\left(r e^{i \theta}\right)\right)\right| d \theta \\
& +\frac{1}{2 \pi} \int_{0}^{2 \pi} \log ^{+}\left|D_{1}\left(\tau^{-1}\left(r e^{i \theta}\right)\right)\right| d \theta .
\end{aligned}
$$

Thus we have for any unit vectors $x, y$ in $\mathfrak{S}$,

$$
\begin{aligned}
\frac{1}{2 \pi} \int_{0}^{2 \pi} & \log ^{+}\left|\left(\left\{I-B_{1}\left(\tau^{-1}\left(r e^{i \theta}\right) I-A-B_{2}\right)^{-1}\right\}^{-1} x, y\right)\right| d \theta+\int_{0}^{r} \frac{n(t)}{t} d t \\
= & \frac{1}{2 \pi} \int_{0}^{2 \pi} \log ^{+}\left|\frac{D_{2}\left(\tau^{-1}\left(r e^{i \theta}\right)\right)}{D_{1}\left(\tau^{-1}\left(r e^{i \theta}\right)\right)}\right| d \theta+\int_{0}^{r} \frac{n(t)}{t} d t \\
\leqq & \frac{1}{2 \pi} \int_{0}^{2 \pi}\left[\log ^{+}\left|D_{2}\left(\tau^{-1}\left(r e^{i \theta}\right)\right)\right|+\log ^{-}\left|D_{1}\left(\tau^{-1}\left(r e^{i \theta}\right)\right)\right|\right] d \theta \\
& +\int_{0}^{r} \frac{n(t)}{t} d t \\
\leqq & \frac{1}{2 \pi}\left[\int_{0}^{2 \pi} \log ^{+}\left|D_{2}\left(\tau^{-1}\left(r e^{i \theta}\right)\right)\right| d \theta+\int_{0}^{2 \pi} \log ^{+}\left|D_{1}\left(\tau^{-1}\left(r e^{i \theta}\right)\right)\right| d \theta\right] \\
\leqq & \sum_{j} \log \left\{1+\mu_{j}\left(B_{1}\left(\lambda_{1} I-A-B_{2}\right)^{-1}\right)\right\} \\
& +\sum_{j} \log \left\{1+\mu_{j}\left(B_{1}\left(\lambda_{2} I-A-B_{2}\right)^{-1}\right)\right\} \\
& +3 \log (3+1 / 2 \delta),
\end{aligned}
$$


where $\lambda_{1}, \lambda_{2} \in\{\lambda: d(\lambda, \sigma(A))=2 \delta\}$, since the inequality $1+t \leqq e^{t}$ for a real number $t$, it follows

$$
\begin{aligned}
& \leqq \sum_{i=1}^{2}\left\|B_{1}\left(\lambda_{i} I-A-B_{2}\right)^{-1}\right\|_{1}+3 \log (3+1 / 2 \delta) \\
& \leqq(2 / \delta)\left\|B_{1}\right\|_{1}+3 \log (3+1 / 2 \delta) .
\end{aligned}
$$

Thus we have, for any unit vectors $x, y$ in $\mathfrak{S}$ and for $\lambda$ with $d(\lambda, \sigma(A)) \geqq 2 \delta$,

$$
\begin{aligned}
& \frac{1}{2 \pi} \int_{0}^{2 \pi} \log ^{+}\left|\left(\left\{I-B_{1}\left(\tau^{-1}\left(r e^{i \theta}\right) I-A-B_{2}\right)^{-1}\right\}^{-1} x, y\right)\right| d \theta \\
& \quad \leqq(2 / \delta)\left\|B_{1}\right\|_{1}+3 \log (3+1 / 2 \delta) .
\end{aligned}
$$

On the other hand by the Poisson-Jensen formula [12, Theorem 1.1, p. 1] we have

$$
\begin{aligned}
\log \mid & \left(\left\{I-B_{1}\left(\tau^{-1}\left(\rho e^{i \varphi}\right) I-A-B_{2}\right)^{-1}\right\}^{-1} x, y\right)\left|-\sum_{k} \log \right| \frac{r\left(z-z_{k}\right)}{r^{2}-\bar{z}_{k} z} \mid \\
= & \frac{1}{2 \pi} \int_{0}^{2 \pi} \log \mid\left(\left\{I-B_{1}\left(\tau^{-1}\left(r e^{i \theta}\right) I-A-B_{2}\right)^{-1} x, y\right) \mid\right. \\
& \times \frac{r^{2}-\rho^{2}}{r^{2}-2 r \rho \cos (\theta-\varphi)+\rho^{2}} d \theta
\end{aligned}
$$

where $z=\rho e^{i \varphi}, 0 \leqq \rho<r$ and $\left\{z_{k}\right\}$ are zeros of the function

$$
\left(\left\{I-B_{1}\left(\tau^{-1}(z) I-A-B_{2}\right)^{-1}\right\}^{-1} x, y\right)
$$

in $|z|<r$. Since $0<\left|r\left(z-z_{k}\right) /\left(r^{2}-\bar{z}_{k} z\right)\right|<1$ and

$$
\frac{r-\rho}{r+\rho} \leqq \frac{r^{2}-\rho^{2}}{r^{2}-2 r \rho \cos (\theta-\varphi)+\rho^{2}} \leqq \frac{r+\rho}{r-\rho},
$$

we have

$$
\begin{aligned}
\log \mid & \left(\left\{I-B_{1}\left(\tau^{-1}\left(\rho e^{i \varphi}\right) I-A-B_{2}\right)^{-1}\right\}^{-1} x, y\right) \mid \\
& \leqq \frac{r+\rho}{r-\rho} \frac{1}{2 \pi} \int_{0}^{2 \pi} \log ^{+}\left|\left(\left\{I-B_{1}\left(\tau^{-1}\left(r e^{i \theta}\right) I-A-B_{2}\right)^{-1}\right\}^{-1} x, y\right)\right| d \theta \\
& \leqq \frac{r+\rho}{r-\rho}\left\{(2 / \delta)\left\|B_{1}\right\|_{1}+3 \log (3+1 / 2 \delta)\right\} .
\end{aligned}
$$

Since $\tau$ is a conformal mapping, therefore we have

$$
\begin{aligned}
\log \| & \left\{I-B_{1}\left(\lambda I-A-B_{2}\right)^{-1}\right\}^{-1} \| \\
& \leqq O(1 / \delta)\left\{(2 / \delta)\left\|B_{1}\right\|_{1}+3 \log (3+1 / 2 \delta)\right\},
\end{aligned}
$$

for $\lambda$ with $d(\lambda, \sigma(A)) \geqq 3 \delta$. Therefore from the already proved inequalities in above, we have 
$\log \left\|(\lambda I-T)^{-1}\right\| \leqq \log (1 / \delta)+O(1 / \delta) \cdot\left\{(2 / \delta)\left\|B_{1}\right\|_{1}+3 \log (3+1 / 2 \delta)\right\}$, for $\lambda$ with $d(\lambda, \sigma(A)) \geqq 3 \delta$.

We put $\gamma(t)=\gamma(t, B)$. Using this function we can write

$$
\log \left\|(\lambda I-T)^{-1}\right\| \leqq \log (1 / \delta)+O(1 / \delta) \cdot\{(2 / \delta) \gamma(1 / \delta)+3 \log (3+1 / 2 \delta)\},
$$

for $\lambda$ with $d(\lambda, \sigma(A)) \geqq 3 \delta$. Then we have for sufficiently small $\delta>0$,

$$
\begin{aligned}
\sup _{d(\lambda, \sigma(A)) \geq 3 \delta} & \log \log \left\|(\lambda I-T)^{-1}\right\| \\
\leqq & \log \log (1 / \delta)+O \log (1 / \delta)+\log (2 / \delta)+\log \gamma(1 / \delta) \\
& +\log \{3 \log (3+1 / 2 \delta)\} .
\end{aligned}
$$

Here, $\log \log (1 / \delta)$ and $\log (1 / \delta)$ are integrable on some interval $(0, \varepsilon]$, let us prove that $\log \gamma(1 / \delta)$ is integrable on $(0, \varepsilon]$. This follows from the assumption, $B \in C_{\omega}$, in fact

$$
\begin{aligned}
\int_{0}^{\varepsilon} \log \gamma(1 / \delta) d \delta & =\int_{1 / \varepsilon}^{\infty} \frac{\log \gamma(t)}{t^{2}} d t \\
& =\int_{1 / \varepsilon}^{\infty} \frac{d \gamma(t)}{\gamma(t) t}-\varepsilon \log \gamma(1 / \varepsilon)+\lim _{t \rightarrow \infty} \frac{\log \gamma(t)}{t},
\end{aligned}
$$

while

$$
\int_{1 / \varepsilon}^{\infty} \frac{d \gamma(t)}{\gamma(t) t} \leqq \sum_{n} \mu_{n}(B) / n
$$

with $\varepsilon \mu_{1}(B)=1$, since $\log n<1+1 / 2+1 / 3+\cdots+1 / n$, we have

$$
\frac{\log \gamma(t)}{t} \leqq \Sigma^{\prime} \mu_{k}(B) / k \leqq \sum_{n} \mu_{n}(B) / n,
$$

where $\Sigma^{\prime}$ denotes the summation over $k$ such that $\mu_{k}(B) \geqq 1 / t$. Therefore we can obtain the following growth condition of the resolvent of $T$ near its spectrum

$$
\int_{0}^{\varepsilon} \log \log M(\delta) d \delta<\infty,
$$

where $M(\delta)=\sup _{d(\lambda, \sigma(A)) \geq 3 \delta}\left\|(\lambda I-T)^{-1}\right\|$.

From the above growth condition of the resolvent of $T$ near its spectrum, by virtue of Theorem 2.2 (in section 2) Theorem in this section was obtained.

CoRollary 3.5. Let $T \in \mathfrak{B}(\mathfrak{S})$ ). If $T-T^{*} \in C_{\omega}(i . e$. , the imaginary part of $T$ belongs to $C_{\omega}$ ) and $\sigma(T)$ is not reduced to a single point, then $T$ has a proper closed hyperinvariant subspace in $\mathfrak{F}$. 


\section{REFERENCES}

[1] N. ARonszajn and K. T. Sмith, Invariant subspace of completely continuous operators, Ann. of Math., 60 (1954), 345-350.

[2] C. Apostol, On the growth of resolvent, perturbation and invariant subspaces, Rev. Roum. Math. Pures et Appl., 16 (1971), 161-172.

[ 3 ] N. Dunford and J. T. Schwartz, Linear operators, Part I, 1958; Part II, 1963, Interscience, New York.

[4] I. C. GoHberg AND M. G. KREIN, Introduction to the theory of linear non-selfadjoint operators, Transl. Math. Mono., Vol. 18, Amer. Math. Soc., 1969.

[5] K. Kitano, Invariant subspaces of some non-selfadjoint operators, Tôhoku Math. J., 20 (1968), 313-322.

[6] B. JA. Levin, Distribution of zeros of entire functions, Transl. Math. Mono., Vol. 5, Amer. Math. Soc., 1964.

[7] N. Levinson, Gap and density theorem, Amer. Math. Soc. Colloq. Publ., Vol. 26, 1940.

[8] Ju. I. LJubič AND V. I. MACAev, On operators with a separable spectrum, Mat. Sb., 56 (98) (1962), 433-468; Amer. Math. Soc. Transl., (2), 47 (1965), 89-129.

[9] V. I. MACAEv, A class of completely continuous operators, Dokl. Akad. Nauk SSSR, 139 (1961), 548-551.

[10] V. I. MACAEV, A method for the estimation of the resolvents of non-selfadjoint operators, Dokl. Akad. Nauk SSSR, 154 (1964), 1034-1037.

[11] F. Riesz AND B. Sz.-NAGY, Functional analysis, Fredrick Unger, New York, 1955.

[12] W. K. Hayman, Meromorphic functions, Oxford Math. Mono., Oxford Univ., London, 1964.

DEPARTMENT OF MATHEMATICS

YAMAGATA UNIVERSITY,

YAMAGATA, JAPAN 
